\title{
Lapurdum
}

LAPURDUM Euskal ikerketen aldizkaria | Revue d'études basques |

Revista de estudios vascos | Basque studies review

$1 \mid 1996$

Numéro I

\section{La langue basque défigurée}

Michel Morvan

\section{OpenEdition}

Journals

Édition électronique

URL : http://journals.openedition.org/lapurdum/1911

DOI : 10.4000/lapurdum.1911

ISSN : 1965-0655

Éditeur

IKER

Édition imprimée

Date de publication : 1 octobre 1996

Pagination : 223-227

ISBN : 2-84127-106-4

ISSN : $1273-3830$

Référence électronique

Michel Morvan, «La langue basque défigurée », Lapurdum [En ligne], 1 | 1996, mis en ligne le 01 septembre 2010, consulté le 29 janvier 2020. URL : http://journals.openedition.org/lapurdum/1911 ; DOI : 10.4000/lapurdum.1911 
Michel MORVAN

\section{LA LANGUE BASQUE DÉFIGURÉE}

“Jacques Auber n'est ni un inconnu ni un néophyte. Issu et diplômé de l'École nationale des langues orientales vivantes, il s'est illustré brillamment dans la rédaction et la publication de plusieurs ouvrages qui sont restés des références en la matière dont Histoire de l'Océan Indien, La langue malgache en 30 familles de mots. Après de nombreuses années passées au Moyen-Orient, à Madagascar puis à Téhéran, Jacques Auber a enseigné l'Histoire pendant une dizaine d'années au lycée Saint-Thomas-d'Aquin à Saint-Jean-de-Luz" (1)

Je ne doute pas un instant que M. Jacques Auber soit un éminent spécialiste de la langue malgache. Je suis évidemment beaucoup plus perplexe lorsqu'à partir de ces connaissances ultramarines il entend retrouver l'origine de l'euskara, rien de moins ! Étant moi-même un modeste paléo-linguiste qui tente de remonter le temps avec les quelques armes dont je dispose, je suis très ouvert à toutes les idées qui peuvent faire avancer la question des origines du basque. Pour autant, il y a néanmoins des limites à ce qu'un chercheur, même bien disposé et à l'esprit ouvert, peut accepter.

Ces limites, l'auteur de La langue basque démasquée ${ }^{(2)}$ les franchit allégrement au mépris le plus absolu des règles les plus élémentaires de la conduite d'une recherche scientifique un tant soit peu acceptable.

Dans l'absolu, l'idée de départ n'a rien en soi qui puisse vraiment choquer. Il s'agit pour l'auteur de comparer des racines très anciennes de certaines langues avec cette autre langue aux couches lexicales très anciennes qu'est le basque. C'est ce que je fais depuis quinze ans. Mais l'application concrète et la méthode utilisée par l'auteur sont catastrophiques, ce qui a pour résultat immédiat de classer d'emblée l'ouvrage de J. Auber aux côtés de ceux d'autres auteurs, dont la liste est assez longue hélas, que l'on a coutume de considérer comme des élucubrations fantaisistes. J'en ai dénoncé quelques-unes dans ma thèse de doctorat ${ }^{(3)}$. Dieu sait pourtant que je ne suis pas un chasseur de sorcières. Je fais tout mon possible pour éviter quelque dogmatisme que ce soit, mais trop c'est trop. Je ne livrerai personne au bûcher, le ridicule suffira.

La méthode est toujours la même : utilisation de références sérieuses pour donner à l'ouvrage inepte un aspect scientifique ou pseudo-scientifique, ce qui d'ailleurs, en l'occurrence, n'empêche pas une absence totale de bibliographie à la fin du livre. 
Un autre "truc", pas toujours volontaire au demeurant, car il y a des illuminés sincères hélas, consiste à mélanger à un ensemble franchement mauvais quelques bonnes équations du genre basque aita "père" = quechua aita "id." = turc ata "id." = eskimo atata "id.". Il est évident qu'il y a là un fonds lexical extrêmement ancien commun à de nombreuses langues du monde. Le problème, c'est que ce sont des faits déjà largement connus des linguistes, et le lecteur risque de croire que c'est une extraordinaire découverte de l'auteur. L'ennui, aussi, avec la plupart des amateurs (et même parfois avec certains universitaires ou chercheurs !), c'est qu'ils ne savent pas faire la part des choses et démêler le bon grain de l'ivraie. Il marrive de recevoir du courrier de correspondants français et étrangers qui ne sont pas nécessairement des spécialistes. Aussi ai-je appris à distinguer les bonnes choses qui parfois peuvent, sans être des travaux scientifiques classiques, se trouver comme des perles rares au milieu d'un ensemble confus. Il n'y a pas si longtemps, j'ai eu la bonne surprise de recevoir une lettre d'un correspondant roumain qui a retenu mon attention. Cet amateur n'avait pas manqué de tomber dans le piège qui consistait à comparer des termes roumains et des termes basques parfaitement superposables pour en déduire une parenté entre les deux langues. Il s'agissait évidemment de termes que les deux langues tenaient du latin, indépendamment l'une de l'autre. Le basque par l'intermédiaire des emprunts au latin, et le roumain parce qu'il est précisément une langue latine. Or, au milieu de ces pseudo-rapprochements, figuraient bien cachés quelques termes roumains non issus du latin, mais du substrat pré-latin de cette langue. Ma réaction fut immédiate. Cela, on ne l'apprend qu'avec l'expérience.

L'autre idée de départ de J. Auber n'est pas absurde non plus dans l'absolu. Le langage humain aurait commencé par des onomatopées. Pourquoi pas ? Mais ce n'est pas, là-encore, une idée originale de l'auteur. Bien d'autres l'ont pensé avant lui. Et à nouveau l'application concrète qui découle de ce postulat de départ est absolument catastrophique, comme on va pouvoir en juger ci-après. On peut en effet parfaitement travailler sur les onomatopées et les termes expressifs communs à plusieurs langues, et il est exact qu'il ne s'agit pas toujours de coïncidences, mais parfois de réelles traces d'un fonds linguistique commun. Le problème, c'est qu'il ne faut pas faire n'importe quoi n'importe comment, qu'il faut savoir exactement où l'on va. Le bateau de M. J. Auber n'est malheureusement qu'un bateau ivre sans boussole.

Je passe sur quelques affirmations dénuées de tout fondement comme par ex. "l'homme de Cro-Magnon ne disposait pas encore d'une véritable langue autre que l'expression artistique, les cris, les gestes et la mimique. Il n'avait pas encore de "discours" à tenir" (p.23). C'est totalement faux évidemment. Les Magdaléniens possédaient un langage parfaitement élaboré et l'auteur oublie, ou plutôt ne sait pas, que les premiers Amérindiens, passés d'Asie en Amérique il y a près de trente mille ans, ont importé leurs langues avec eux puisqu'on en retrouve des traces de chaque côté du Détroit de Behring. L'auteur confond sans doute Cro-Magnon, type Homo Sapiens déjà identique à nous avec 
l'Homo Erectus ou l'Homo Habilis dont le langage était effectivement très primitif, en développement naissant avec une très faible palette de sons possibles (cerveau plus petit, larynx placé encore trop haut, etc.). Et l'auteur d'ajouter comme pour mieux enfoncer le clou : “...Mais dès que l'existence d'un langage digne de ce nom leur fut connu (probablement à l'approche de la Méditerranée) ils l'adoptèrent comme les peuples frustes se jettent sur les nouveautés pratiques. Ainsi firent de notre temps les Polynésiens pour la casquette et le vélo..." (sic).

On demeure interdit devant tant de naïveté. Quant aux "peuples frustes", je laisse à l'auteur la responsabilité de cette expression. Ce ne sont pas ces peuples-là qui ont inventé au $\mathrm{XX}^{e}$ siècle les camps de concentration, les chambres à gaz et autres monstruosités. Et nous en arrivons maintenant au feu d'artifice des mots. Bien sûr, l'auteur prend soin de dénoncer les travaux de J. Lemoine qui fait venir à tort des mots basques "autochtones" de mots latins, mais c'est pcur nous asséner aussitôt à son tour d'énormes âneries comme celle qui consiste à faire provenir le basque oin "pied" du français bottine (sic, p. 25 et 152) ou le basque zenbaki "numéro", du français abaque!! On est là en plein délire. Le terme zenbaki est un composé de zein "quel, combien" et de bat "un, unité". Quant à oin, on voit le genre de "méthode" utilisé par l'auteur: le mot français $b O t t I N e$ contient les trois lettres $o, i$ et $n$, alors pourquoi n'aurait-il pas donné le basque oin "pied" ? On se demande si on rêve. Même les pseudo-rapprochements de J. Lemoine ne parviennent pas à un tel degré d'absurdité. Le français bottine apparaît comme dérivé de botte en 1367. Le basque oin "pied" doit lui être antérieur de quelques centaines, voire de quelques milliers d'années! C'est un vieux terme du fonds basque autochtone. En l'état actuel des connaissances, un seul rapprochement qui pourrait être troublant par rapport à ce terme a pu être découvert tout récemment en Eurasie par votre serviteur dans la langue mongole, où onie désigne une sorte d'étai de soutien (= jambe) de la yourte. Et ce n'est qu'une hypothèse de travail.

L'auteur n'hésite pas, dans ses comparaisons, à inventer des mots qui n'existent pas, comme par ex. le japonais ogi "pain"(p. 24). Je n'interdis à personne de comparer le terme basque ogi "blé, pain" avec un terme japonais, encore faut-il au moins le comparer avec le terme réel qui est mugi. On posera alors l'hypothèse cette fois (mais n'étant rien d'autre qu'une hypothèse) de la chute d'un $m$ - initial, etc. Là commencerait un travail déjà plus intéressant et plus méthodique.

La méconnaissance complète qu'a l'auteur de l'histoire des mots basques lui fait, bien entendu, comparer des termes dont la parenté est impossible. C'est le cas de haitz "arbre"qu'il compare (p. 26) au mélanésien kasu, au malgache hazo et à l'hébreu etz "id.". Il ignore évidemment le fait décisif qui est que haitz "arbre" n'apparaît en basque que tardivement (attesté au milieu du XIX ${ }^{e}$ siècle) ${ }^{(4)}$ et n'est autre qu'une contraction de haritz "chêne", cet arbre étant considéré comme "l'arbre par excellence", d'où le sens général "arbre" pris par la forme contractée en question. À ne pas confondre avec -haitz dans zuhaitz "arbre" qui est un composé de zur "bois" et de gaitz "grand" attesté dès le X' siècle. 
Voilà ce qui arrive lorsqu'on ignore complètement l'historique de l'évolution d'un mot.

Suit le corps du "livre" proprement dit qui n'est qu'une longue série de tableaux où règne l'arbitraire le plus absolu, avec évidemment de-ci de-là quelques rapprochements qui tombent juste, car il est statistiquement inévitable que vu le nombre de listes et de tableaux alignés par l'auteur le taux de $100 \%$ d'erreurs soit évité ! Toute la théorie de l'auteur se résume concrètement à ce postulat : les mots viennent d'onomatopéees dont la consonne centrale est la colonne vertébrale. À partir de là, on trouve mis côte à côte tous les termes possibles et imaginables. Le basque sudur "nez" se retrouve apparenté au basque otan "boussole", simplement parce que ces deux termes ont une dentale comme consonne centrale !! C'est à se taper le derrière par terre ! L'exemple de la page 33 où bazka "pacage, nourriture" est associé à bazkari "repas, dîner" montre là encore l'ignorance de l'évolution historique. Le terme bazkari n'apparaît qu'en $1686^{(3)}$ et vient par contraction d'une forme plus ancienne barazkari ${ }^{\circ}$ dans laquelle le premier élément est baratze "jardin potager". Page 39, le terme begi "œil" est associé à d'autres termes débutant par une consonne labiale. Pas de chance pour l'auteur, hélas, car le $b$-initial de ce mot n'appartient pas à la racine qui est "eg-. et que l'on retrouve dans egun "jour", eguzki "soleil", egia "vérité".

On pourrait continuer comme cela jusqu'à la fin de l'ouvrage, mais je crois que cela n'en vaut vraiment pas la peine. Tout est à l'avenant. Il n'y a pas une page où ne figure quelque énormité, et même souvent plusieurs. Même un emprunt au latin archi-connu comme ahate "canard", du lat. anatem "id." est ignoré par l'auteur qui le fait venir du basque aho "bouche"!! Et je passe sur des choses comme oti "sauterelle" qui est présenté par l'auteur comme une déformation (sic) du français sauterelle!!

Que dire, que faire devant un élaborat de ce genre ? On est tellement abasourdi, on n'en croit pas ses yeux. Faut-il en pleurer ou en rire ? À vous de choisir. 


\section{NOTES}

1. E. Dupré-Moretti, Préface à La langue basque démasquée de J. Auber, p. 11.

2. J. Auber, La langue basque démasquée, Ikuska, 1995.

3. M. Morvan, Les origines linguistiques du basque, thèse, Bordeaux, 1992, p. 22-29.

4. Cf. J.-M. Hiribarren, Eskaldunac, Bayonne, 1853, p. 87.

5. Cf. B. Gasteluçar, Eguia Catholicac, Pau, 1686, p. 124.

6. Cf. B. Dechepare, Linguae Vasconum Primitiae, Bordeaux, 1545, p. 25. 